\section{Enfrentamiento de pacientes pediátricos con várices esofágicas}

\author{
SOFÍA VERDAGUER D. ${ }^{1, a}$, JUAN CRISTÓBAL GANA A. ${ }^{2}$
}

\section{Management of pediatric patients with esophageal varices}

Background: There is a paucity of good quality research about the diagnosis of esophageal varices and the prophylaxis and treatment of variceal bleeding in pediatric patients with portal hypertension There is little consensus and practically no evidence-based approach about the management of these patients. Aim: To describe the behavior and preferences of pediatric gastroenterologists in Chile in the management of portal hypertension in children. Material and Methods: An online survey was sent to Chilean pediatric gastroenterologists, with questions evaluating the physicians' approaches to screening of esophageal varices in children with portal hypertension, and their preferred methods of prophylaxis and initial management of variceal bleeding. Results: Thirty five of 69 contacted physicians answered the survey (51\%). Twenty nine pediatric gastroenterologists (83\%) screen for esophageal varices in patients with clinical evidence of portal hypertension, and $12(34 \%)$ in every patient with chronic liver disease. Twenty eight respondents (80\%) use primary prophylaxis, mainly beta blockers. Octreotide, proton pump inhibitors and endoscopy are the most common practices in the initial management of an esophageal varix bleed. The methods mostly used as secondary prophylaxis are band ligation and beta blockers. In the case of recurrent hemorrhage, besides band ligation, management with Transjugular Intrahepatic Portosystemic Shunt (TIPS) and hepatic transplantation are more likely. Conclusions: Even though most pediatric gastroenterologists in this survey are inclined to offer endoscopic screening of esophageal varices and prophylaxis to patients with portal hypertension, this is not a universal behavior. There are different approaches mainly in the election of secondary prophylaxis and the initial management of variceal bleeding.

(Rev Med Chile 2016; 144: 879-885)

Key words: Diagnosis; Esophageal and Gastric Varices; Hypertension, Portal; Pediatrics; Primary Prevention; Secondary Prevention.
1Escuela de Medicina, Pontificia Universidad Católica de Chile, Santiago, Chile. 2Departamento de Gastroenterología y Nutrición Pediátrica. División de Pediatría, Escuela de Medicina, Pontificia Universidad Católica de Chile, Santiago, Chile.

anterna.

Sin fuentes de apoyo financiero

Recibido el 18 de noviembre de 2015, aceptado el 11 de junio de 2016.

Correspondencia a: Juan Cristóbal Gana A. Lira 85, 5to piso. Santiago, Chile jcgana@gmail.com

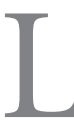
a hemorragia gastrointestinal por várices esofágicas es una complicación grave de la hipertensión portal secundaria a daño hepático crónico u obstrucción de vena porta en la población pediátrica. Su incidencia en niños con atresia biliar va desde $17 \%$ a $19 \%$ en un período de 5 a 10 años ${ }^{1-3}$ y es de $50 \%$ en niños que sobreviven más de 10 años sin un trasplante hepático ${ }^{4}$. La mortalidad asociada descrita es de $2,5 \%$ a $20 \% \%^{2,5-7}$.
A pesar de su importancia, la mayor parte de la evidencia sobre el tratamiento de esta patología proviene de pacientes adultos.

En adultos, la prevalencia de várices esofágicas es de $50 \%$ en los pacientes cirróticos, y su presencia se correlaciona con el grado de enfermedad hepática, siendo más prevalentes en daño más avanza$\mathrm{do}^{8}$. Se ha estimado que el riesgo de hemorragia en un paciente que presenta várices esofágicas es 
de 25\%-35\% en dos años; la presencia de mayor tamaño de las várices y la asociación de "puntos rojos" evidenciables en la endoscopia digestiva alta (EDA) confieren un mayor riesgo ${ }^{9,10}$. Se ha demostrado beneficio en la población adulta del uso de profilaxis primaria con beta-bloqueadores y ligadura endoscópica de várices ${ }^{11}$.

En niños existe un menor número de publicaciones sobre este tema, la mayoría son reportes de casos y con tamaño muestral pequeño. Estudios recientes han demostrado que ciertos parámetros clínicos y endoscópicos en niños con várices podrían predecir el riesgo de hemorragia variceal a lo largo del tiempo ${ }^{12}$. Existe sólo un estudio randomizado que demuestra que en pacientes seleccionados la terapia endoscópica como profilaxis primaria disminuye el riesgo de hemorragia ${ }^{5}$. Sobre profilaxis secundaria, existen estudios observacionales que sugieren que la ligadura endoscópica de várices puede ser una medida efectiva y segura a largo plazo ${ }^{14}$. A pesar de que es posible diagnosticar las várices en pacientes pediátricos y existen primeras aproximaciones para estimar el riesgo de que éstas sangren, no existe evidencia sólida que respalde el tratamiento preventivo de la hemorragia.

La hemorragia digestiva de origen variceal confiere un aumento del riesgo de morbi-mortalidad tanto en el episodio agudo como a largo plazo. En adultos, existen guías internacionales para el tratamiento de la hemorragia digestiva alta de origen variceal, en unidades de alta complejidad que incluyen el uso de drogas vasoactivas como la Terlipresina u Octreótido, antibióticos, inhibidores de bomba de protones, transfusiones y tratamiento endoscópico ${ }^{8,15}$. En niños, debido a la falta de estudios clínicos, hay una gran variabilidad de tratamientos entre los centros y muchos son extrapolados de la evidencia en adultos ${ }^{16,17}$.

Al no existir una conducta basada en evidencia en esta enfermedad, el tratamiento de un paciente con hipertensión portal y sus complicaciones puede variar según su médico tratante. El objetivo de este trabajo es, mediante una encuesta, conocer las prácticas clínicas actuales que realizan los gastroenterólogos pediátricos en Chile respecto al diagnóstico de várices esofágicas, la indicación de profilaxis primaria, secundaria y el tratamiento de la hemorragia por várices esofágicas en niños.

\section{Métodos}

Se desarrolló la traducción y adaptación de una encuesta realizada previamente en Estados Unidos y Canadá15,16, de la cual se obtuvieron las autorizaciones respectivas. Ésta evalúa la aproximación de los especialistas respecto al screening de várices esofágicas (estudio diagnóstico antes del episodio de hemorragia), profilaxis primaria (tratamiento preventivo de la hemorragia variceal), manejo agudo de la hemorragia variceal y profilaxis secundaria (tratamiento preventivo luego del primer episodio de hemorragia variceal). Se contactó a todos los gastroenterólogos pediátricos chilenos vía email y se les solicitó que respondieran la encuesta en forma anónima a través de un servidor encriptado y protegido (Red Cap). En total, se enviaron 4 solicitudes entre el 2012 y 2013.

La encuesta consiste en 13 preguntas que fueron testeadas con antelación entre los investigadores para asegurar su claridad. El cuestionario consta de 3 secciones. La primera, con 3 preguntas sobre la experiencia del médico en el ámbito de la hepatología pediátrica y el manejo de niños con hipertensión portal. La segunda, con 7 preguntas que van en relación con el tratamiento del paciente pediátrico con hipertensión portal. Finalmente, la tercera sección consta de 3 preguntas sobre el enfrentamiento respecto a los riesgos y beneficios del procedimiento endoscópico, evaluado según escalas de Likert de 5 niveles. El tiempo estimado para completar la encuesta fue de $10 \mathrm{~min}$.

Con los datos se calculó el porcentaje de respuesta para cada pregunta, presentando la información a través de tablas.

\section{Resultados}

Luego de contactar a 69 gastroenterólogos pediátricos, se obtuvieron 33 respuestas vía online y 2 por documento escrito, lo que corresponde a $51 \%$ de los encuestados. Siete $(20 \%)$ consideraron que la hepatología pediátrica es su área de interés más importante. Los médicos tenían de experiencia un promedio de 12,5 años trabajando como gastroenterólogos pediátricos y realizaban seguimiento clínico a 7,3 pacientes en promedio con hipertensión portal probable o confirmada.

Respecto al screening para várices esofágicas, 29 médicos $(83 \%)$ lo realizan en ciertas condiciones, 
Tabla 1. Screening de várices: tipo de paciente, método e intervalo

\begin{tabular}{|lr|}
\hline & Número de encuestados (\%) \\
\hline Tipo de paciente al que realiza screening & $12(34)$ \\
Todos los con daño hepático crónico & $14(40)$ \\
Todos los con evidencia de cirrosis & $26(74)$ \\
Con daño hepático y esplenomegalia & $22(63)$ \\
Con daño hepático y trombocitopenia & $22(63)$ \\
Con daño hepático y evidencia ecográfica de colaterales portosistémicas & $31(89)$ \\
Método de screening & $8(23)$ \\
Endoscopia & 1 \\
Ecografía & $(3)$ \\
Scanner & 0 \\
Esofagograma con bario & $(0)$ \\
Intervalo entre procedimientos de screening en paciente sin várices & $8(24)$ \\
6 meses & $23(70)$ \\
12 meses & 1 \\
24 meses & $(3)$ \\
\hline 24 meses & 1 \\
No responde & $(3)$ \\
\hline
\end{tabular}

que incluyen: $12(34 \%)$ a todo paciente con daño hepático crónico, 26 (74\%) a pacientes con daño hepático asociado a esplenomegalia, 22 (63\%) a pacientes con daño hepático y trombocitopenia, $22(63 \%)$ a pacientes con daño hepático y evidencia ecográfica de colaterales portosistémicas, y 14 (40\%) a pacientes con evidencia de cirrosis. Seis médicos (7\%) no realizan screening de várices en forma rutinaria a sus pacientes.

El siguiente caso clínico fue presentado en el cuestionario, basado en la publicación previa, sobre screening y profilaxis primaria: "Paciente de 8 años con atresia de vías biliares, el que fue sometido a cirugía de Kasai (portoenterostomía) en el período de lactante menor. Evolución favorable clínicamente, a excepción del desarrollo de hipertensión portal. Presenta crecimiento y desarrollo normales, sin historia de complicaciones de su patología hepática. Resultados de laboratorio incluyen: plaquetas $65.000 / \mathrm{mm}^{3}$, albúmina $3,3 \mathrm{~g} /$ dl, bilirrubina total $1,6 \mathrm{mg} / \mathrm{dl}$, INR 1,1 (tiempo de protrombina 13,2 s). ¿Realizaría Ud. procedimientos de screening para várices esofágicas?” A lo anterior, 33 (94\%) respondieron que sí realizarían procedimientos para screening de várices esofágicas: 31 (89\%) usando endoscopia. La Tabla 1 muestra el intervalo de tiempo preferido por los médicos para repetir el método de screening en caso de no encontrar várices.
En el caso de encontrar várices esofágicas, 28 $(80 \%)$ realizarían profilaxis primaria, $25(71 \%)$ utilizando beta-bloqueadores, 11 (31\%) usando ligadura endoscópica y $3(9 \%)$ escleroterapia (Tabla 2).

En el tratamiento de la hemorragia gastrointestinal con compromiso hemodinámico en pacientes con hipertensión portal, las medidas iniciales más utilizadas por los encuestados son: octreótido por 23 médicos (83\%), inhibidores de bomba de protones por $28(80 \%)$, y endoscopia por $21(60 \%)$ (Tabla 3$)$. En el mismo caso anterior, $3(9 \%)$ contestaron que no realizan endoscopia en estos pacientes, 7 (20\%) la realizan siempre en todos los pacientes, 25 (71\%) sólo en pacientes hemodinámicamente estables. Ninguno la realiza sólo en pacientes hemodinámicamente inestables.

Tabla 2. Uso reportado de métodos de profilaxis primaria para hemorragia variceal

\begin{tabular}{|c|c|}
\hline & $\begin{array}{l}\text { Número de encuestados } \\
\text { que utiliza el método (\%) }\end{array}$ \\
\hline Beta bloqueadores & $25(71)$ \\
\hline Escleroterapia & $3 \quad(9)$ \\
\hline Ligadura & $11(31)$ \\
\hline Ninguno & $7(20)$ \\
\hline
\end{tabular}


Tabla 3. Uso reportado de medidas incluidas en el tratamiento inicial en un niño con hemorragia gastrointestinal aguda

\begin{tabular}{|lc|}
\hline Sonda nasogástrica & $\begin{array}{c}\text { Número de encuestados que utiliza } \\
\text { el método (\%) }\end{array}$ \\
\hline Antibióticos endovenosos & $11(31)$ \\
Vasopresina & $15(43)$ \\
Somatostatina & $6(17)$ \\
Octreótido & $4(11)$ \\
Sonda de oclusión (sonda Sengstaken-Blakemoretube o sonda Minnesota) & $29(83)$ \\
Endoscopia & $3(9)$ \\
Inhibidores de bomba de protones & $21(60)$ \\
\hline
\end{tabular}

Tabla 4. Uso reportado de métodos de profilaxis secundaria

\begin{tabular}{|c|c|c|}
\hline & \multicolumn{2}{|c|}{ Número de encuestados que usa el método de profilaxis secundaria (\%) } \\
\hline & $\begin{array}{l}\text { Luego del primer episodio } \\
\text { de hemorragia variceal }\end{array}$ & $\begin{array}{l}\text { Luego de hemorragia } \\
\text { variceal recurrente }\end{array}$ \\
\hline Beta bloqueadores & $21(60)$ & $13(37)$ \\
\hline Escleroterapia & $5(14)$ & 1 (3) \\
\hline Ligadura & $34(97)$ & $19(54)$ \\
\hline TIPS & $2(6)$ & $15(43)$ \\
\hline Shunt quirúrgico & $0 \quad(0)$ & $11(31)$ \\
\hline Trasplante & 3 (9) & $16(46)$ \\
\hline
\end{tabular}

TIPS: Derivación portosistémica intrahepática transyugular.

Para indagar sobre el uso de profilaxis secundaria, se utilizó el mismo caso del niño de 8 años con cirugía por atresia de la vía biliar, que evoluciona con hemorragia por várices esofágicas con compromiso hemodinámico, que luego de medidas iniciales que incluyeron transfusión de glóbulos rojos se encuentra estable. La medida de profilaxis secundaria preferida por la mayoría (35 médicos [97\%]) es la ligadura, y en segundo lugar, los beta-bloqueadores, que serían indicados por $21(60 \%)$. Ninguno recomendaría un shunt quirúrgico luego de un episodio de hemorragia. En el caso de hemorragia variceal recurrente, la ligadura sigue siendo la primera opción, elegida por 19 (54\%), 16 (46\%) indicaría un trasplante y 15 (43\%) indicaría TIPS (Derivación portosistémica intrahepática transyugular) (Tabla 4).

En el caso en que un paciente con hipertensión portal viva alejado de un centro terciario (siendo necesario transporte aéreo), 28 médicos (80\%) cambiarían sus prácticas frente al tratamiento de los pacientes con hipertensión portal. Diez de ellos (29\%) modificaría el screening, 17 (48\%) la profilaxis primaria con ligaduras o beta-bloqueadores, y $5(14 \%)$ cambiaría su indicación de profilaxis secundaria.

Sobre los riesgos de realizar screening para várices, a 19 (54\%) encuestados no les preocupa la posibilidad que la endoscopia produzca hemorragia, mientras que 7 (20\%) sí lo considera importante. A 26 (74\%) médicos no les preocupa la posibilidad que la endoscopia produzca una perforación, a $4(12 \%)$ sí les preocupa este riesgo. La necesidad de utilizar una vía venosa o la posibilidad de complicaciones por la anestesia le preocupa a $13(34 \%)$ médicos y a 4 médicos 
Tabla 5. Riesgo aceptable para indicar una endoscopia en un paciente con hipertensión portal

\begin{tabular}{|c|c|c|}
\hline $\begin{array}{l}\text { Riesgo de } \\
\text { hemorragia o } \\
\text { perforación }\end{array}$ & $\begin{array}{l}\text { Número de encuestados } \\
\text { que estaría dispuesto a realizar } \\
\text { la endoscopia (\%) }\end{array}$ & $\begin{array}{c}\text { Número de encuestados que estaría dispuesto } \\
\text { a realizar la endoscopia si hubiese } \\
\text { tratamiento para várices grandes (\%) }\end{array}$ \\
\hline 1 en 100.000 & $1 \quad(3)$ & $2(6)$ \\
\hline 1 en 10.000 & $5(14)$ & $3 \quad(9)$ \\
\hline 1 en 1.000 & $14(40)$ & 11 (31) \\
\hline 1 en 100 & $13(37)$ & $12(34)$ \\
\hline 1 en 10 & $1 \quad(3)$ & $6(17)$ \\
\hline No responde & $1 \quad(3)$ & $1 \quad(3)$ \\
\hline
\end{tabular}

(12\%) respectivamente. A 19 (54\%) no les parece preocupante usar una vía venosa en el paciente ni la posibilidad de complicaciones por la anestesia.

Sólo 1 participante $(3 \%)$ no considera un beneficio importante del screening endoscópico de várices el ser capaz de informarle al paciente que existe sólo un riesgo bajo o nulo de hemorragia por várices al no encontrar várices o várices pequeñas. En contraste, 29 (83\%) sí lo consideran un beneficio importante. Todos los encuestados consideran que la posibilidad de encontrar várices grandes, y por lo tanto ser capaz de informarle al paciente que hay un alto riesgo de hemorragia por várices, es un beneficio importante del screening. $31(91 \%)$ médicos consideran la posibilidad de encontrar várices grandes y por lo tanto ofrecerle un tratamiento profiláctico al paciente un beneficio importante, mientras que a $1(3 \%)$ no le parece sustancial.

El último ítem se refiere al riesgo de hemorragia o perforación que el médico solicitante está dispuesto a aceptar para realizar una endoscopia en un paciente con hipertensión portal (Tabla 5).

\section{Discusión}

Los resultados de esta encuesta muestran la realidad sobre la práctica de la mayoría de los gastroenterólogos pediátricos en Chile, en el diagnóstico y tratamiento de las várices esofágicas en niños, información no conocida previamente.

La mayoría de los gastroenterólogos pediátricos chilenos realiza screening de várices esofágicas en niños con daño hepático y alguna evidencia de hipertensión portal (esplenomegalia, tromboci- topenia o colaterales portosistémicas), siendo la endoscopia el método más utilizado.

Entre los encuestados, la mayoría utiliza algún método de profilaxis primaria para la hemorragia variceal, principalmente los beta-bloqueadores y luego la terapia endoscópica con ligadura. Ambos métodos han demostrado ser eficientes en la prevención de hemorragia por várices esofágicas en adultos en numerosos estudios controlados, $y$ su recomendación profiláctica es de rutina, no así en pediatría. Debe tenerse especial cuidado con el uso de beta-bloqueo en pediatría, ya que los niños utilizan el aumento de su frecuencia cardíaca como principal mecanismo de compensación hemodinámica frente a la hipovolemia y éste puede estar limitado con el uso de estos medicamentos, a diferencia de los adultos, que lo realizan a través de la vasoconstricción. Además, la farmacocinética y posibles efectos adversos pueden variar según el rango etario. El uso de beta-bloqueadores debe ser mejor estudiado, sobretodo en niños menores, antes de su recomendación profiláctica universal.

Entre las respuestas que presentaron mayor dispersión se encuentra el tratamiento inicial de la hemorragia gastrointestinal aguda. Esto demuestra que la falta de estudios de buena calidad y consensos lleva a la heterogeneidad de la práctica clínica. El contar con adecuada evidencia es de gran importancia, especialmente en casos de una emergencia como lo es una hemorragia variceal. Por otro lado, $83 \%$ y $80 \%$ usaría Ocreótido e inhibidores de ácido respectivamente. Estas medidas utilizadas por la mayoría de los encuestados estarían apoyadas por los reportes de casos pediátricos y estudios de pacientes adultos.

En cuanto a la profilaxis secundaria, la liga- 
dura endoscópica de várices es utilizada por casi la totalidad de los encuestados, y algo más de la mitad usaría beta-bloqueadores. Por otro lado, hubo también bastante dispersión en las respuestas respecto a la profilaxis secundaria en hemorragia variceal recurrente. Cobran mayor importancia, en este escenario, el uso TIPS y el trasplante hepático, aunque ninguna de estas medidas es preferida por todos los encuestados.

A pesar de que $94 \%$ de los encuestados realizaría screening de várices esofágicas en un paciente con hipertensión portal, sólo $80 \%$ tomaría una conducta terapéutica activa y ofrecería profilaxis primaria en caso de que el paciente presente várices. Esto es concordante con respecto a que $100 \%$ de los encuestados considera un beneficio importante el conocer el hallazgo de várices grandes en la endoscopia y así transmitirles al paciente y su familia el alto riesgo de hemorragia variceal. Por otro lado, existe una tendencia a aceptar un riesgo de hemorragia o perforación por la endoscopia en balance a ofrecer un tratamiento para várices grandes. El contar con un método probado y confiable de profilaxis primaria entonces respaldaría la indicación de endoscopia como método de screening.

Al comparar esta encuesta con las realizadas en Estados Unidos en el 2004 (30 participantes) ${ }^{16}$, y en Canadá en el 2011 (47 participantes) ${ }^{15}$, en todos los lugares, se reporta que más de la mitad de los médicos encuestados realiza screening de várices, pero el rango va desde $63 \%$ en Estados Unidos, $70 \%$ en Canadá y $83 \%$ en Chile. La endoscopia es el método preferido por todos los centros. Así mismo, sólo 58\% indica profilaxis primaria en Canadá versus $84 \%$ y $80 \%$ en Estados Unidos y Chile respectivamente. Además, el método más usado por los gastroenterólogos de Canadá es la ligadura endoscópica de várices, a diferencia de los otros encuestados de Chile y Estados Unidos en que los beta-bloqueadores son los más utilizados. En cuanto a profilaxis secundaria, en todos los centros hay una disminución importante del uso de beta-bloqueadores respecto a la profilaxis primaria, especialmente en Canadá. Además destaca el uso de la escleroterapia como alternativa de tratamiento, donde la literatura en pediatría es escasa y en adultos ha sido desplazada por ligadura debido a sus mejores resultados y menores complicaciones. Una excepción serían los pacientes bajo los 10 kilos de peso, en los que no es posible realizar ligadura y la única terapia endoscópica disponible sería la escleroterapia. Dentro de las similitudes entre las tres encuestas, están el uso de endoscopia digestiva alta y octreótido en el episodio de hemorragia variceal aguda (todos sobre $80 \%$ ), y que la amplia mayoría usa la ligadura endoscópica de várices para profilaxis secundaria.

Dentro de las limitaciones de este estudio, se encuentra el porcentaje incompleto de respuesta de los encuestados, lo que podría significar un sesgo. A pesar de mostrar la conducta a seguir en cada caso, no se indagó sobre las razones del actuar médico. Debido al carácter anónimo de la encuesta, no hay información sobre el lugar de trabajo de los médicos ni el grado de complejidad de los centros de atención o las diferencias en la disponibilidad de recursos, que pueden ser factores que influyan en las indicaciones médicas.

La importancia de los datos aquí obtenidos radica en las diferencias observadas en el tratamiento de los pacientes pediátricos con várices esofágicas. No hay una conducta clara en el actuar de los especialistas, especialmente con respecto a la profilaxis primaria y secundaria y el tratamiento agudo de la hemorragia gastrointestinal en pacientes con hipertensión portal, lo que ocurre tanto a nivel nacional como internacional al comparar con estudios símiles de Canadá y Estados Unidos. La diferencia en el manejo de estas patologías probablemente conducirá a resultados disímiles. Es por esto que resulta esencial contar con estudios de buena calidad que permitan aclarar cuál es la mejor conducta en prevención y tratamiento de estos casos, y así mejorar el pronóstico de nuestros pacientes.

\section{Referencias}

1. Miga D, Sokol RJ, Mackenzie T, Narkewicz MR, Smith D, Karrer FM. Survival after first esophageal variceal hemorrhage in patients with biliary atresia. J Pediatr 2001; 139 (2): 291-6.

2. van Heurn LW, Saing H, Tam PK. Portoenterostomy for biliary atresia: Long-term survival and prognosis after esophageal variceal bleeding. J Pediatr Surg 2004; 39 (1): 6-9.

3. Kobayashi A, Itabashi F, Ohbe Y. Long-term prognosis in biliary atresia after hepatic portoenterostomy: analysis of 35 patients who survived beyond 5 years of age. J Pediatr 1984; 105 (2): 243-6. 
4. Toyosaka A, Okamoto E, Okasora T, Nose K, Tomimoto Y. Outcome of 21 patients with biliary atresia living more than 10 years. J Pediatr Surg 1993; 28 (11): 1498-501.

5. Goncalves ME, Cardoso SR, Maksoud JG. Prophylactic sclerotherapy in children with esophageal varices: longterm results of a controlled prospective randomized trial. J Pediatr Surg 2000; 35 (3): 401-5.

6. Mitra SK, Kumar V, Datta DV, Rao PN, Sandhu K, Singh GK, et al. Extrahepatic portal hypertension: a review of 70 cases. J Pediatr Surg 1978; 13 (1): 51-7.

7. Webb LJ, Sherlock S. The aetiology, presentation and natural history of extra-hepatic portal venous obstruction. Q J Med 1979; 48 (192): 627-39.

8. García-Tsao G, Sanyal AJ, Grace ND, Carey W. Prevention and management of gastroesophageal varices and variceal hemorrhage in cirrhosis. Hepatology 2007; 46 (3): 922-38.

9. The North Italian Endoscopic Club for the Study and Treatment of Esophageal Varices. Prediction of the first variceal hemorrhage in patients with cirrhosis of the liver and esophageal varices. A prospective multicenter study. N Engl J Med 1988; 319 (15): 983-9.

10. Gores GJ, Wiesner RH, Dickson ER, Zinsmeister AR, Jorgensen RA, Langworthy A. Prospective evaluation of esophageal varices in primary biliary cirrhosis: development, natural history, and influence on survival. Gastroenterology 1989; 96 (6): 1552-9.

11. Triantos CK, Burroughs AK. Prevention of the devolopment of varices and fist portal hypertensive bleeding episode. Best Pract Res Clin Gastroenterol 2007; 21 (1): 31-42.

12. Duché $\mathrm{M}$, Ducot B, Ackermann O, Jacquemin E, Bernard O. Progression to high-risk gastroesophageal varices in children with biliary atresia with low-risk signs at first endoscopy. Journal of pediatric gastroenterology and nutrition 2015; 60 (5): 664-8.

13. Duche M, Ducot B, Ackermann O, Baujard C, Chevret L, Frank-Soltysiak M, et al. Experience with Endoscopic Management of High-Risk Gastroesophageal Varices, with and Without Bleeding, in Children with Biliary Atresia. Gastroenterology 2013; 145 (4): 801-7.

14. Kang KS, Yang HR, Ko JS, Seo JK. Long-term outcomes of endoscopic variceal ligation to prevent rebleeding in children with esophageal varices. Journal of Korean medical science 2013; 28 (11): 1657-60.

15. García-Tsao G, Bosch J, Groszmann RJ. Portal hypertension and variceal bleeding unresolved issues. Summary of an American Association for the study of liver diseases and European Association for the study of the liver single-topic conference. Hepatology 2008; 47 (5): 1764-72.

16. Schneider B. Approaches to the management of pediatric portal hypertension: results of an informal survey. Portal Hypertension in the 21st Century 2004; 167-72.

17. Gana JY. Variation of care in children with esophageal varices: a study of physicians', patient's, and families' approaches and attitudes. J Pediatr Gastroenterol Nutr 2011; 52 (6): 751-5. 